\title{
FAMILIA Y ADOLESCENCIA EN COSTA RICA. EL CASO DE LAS FAMILIAS EXPULSORAS
}

\section{FAMILY AND ADOLESCENSE IN COSTA RICA. THE CASE OF FAMILIES THAT THROW THEIR CHILDREN INTO THE STREET}

\author{
Olga Prieto Cruz*
}

"Ahí donde termina la familia, empieza la calle".

\section{RESUMEN}

En el presente artículo se realiza un análisis de la problemática familiar que induce a los miembros menores de edad a buscar la calle, conllevando a trabajar con el concepto de "familias expulsoras". Para ello se tiene en consideración cómo las condiciones sociales y económicas vulnerables en que se encuentran estas familias, afectan su integración y por ende, su rol social. Se hace una caracterización de estas familias, de manera que esto pueda contribuir en la formulación de políticas sociales pertinentes.

PALABRAS CLAVE: FAMILIA * ADOLESCENTE * SOCIEDAD * CONDICIONES ECONÓMICAS *
CONDICIONES SOCIALES

\section{ABSTRACT}

In this article it is making an analysis of the family problems that induces the minor's family members to look for the street. This means that working with the concept of families that throw their children into the street. This takes into consideration how the vulnerable social and economic conditions in which these families, they are affect their integration and therefore, its social role. A characterization of these families is made so that this can contribute to the formulation of relevant social policies.

KEYWORDS: FAMILY * ADOLESCENCE $*$ SOCIETY $*$ ECONOMIC CONDITIONS $*$ SOCIAL CONDITIONS

\footnotetext{
* Escuela de Sociología de la Universidad de Costa Rica (UCR). prietocruz.olga@gmail.com

1 Frase recopilada por funcionarios de Casa Alianza de México, en su trabajo con niños en la calle. Frase dicha por César, niño mexicano de 10 años de edad, s.f.
} 


\section{INTRODUCCIÓN}

Como punto de partida en el presente trabajo, se tiene el paradigma de protección integral de las personas menores de edad que se consigna en la Convención Internacional sobre los Derechos de Niño de las Naciones Unidas (2006) y en el Código de la Niñez y la Adolescencia en Costa Rica (2001), en los cuales se reconoce a las personas menores de edad $y$, particularmente, a los y las adolescentes como ciudadanos, productores de cultura y actores estratégicos relevantes en la construcción del proceso de su desarrollo integral, tanto a nivel personal, como a nivel social y cultural.

Con base en este, se trata de hacer un abordaje de la problemática de la situación familiar de las personas adolescentes que se encuentran en condición de calle ${ }^{2}$, con un enfoque que considere los aspectos que pueden afectar la calidad de vida de las familias. En esto se incluye el impacto no solo en los factores relacionales y económicos, sino también, en los factores del entorno que inciden en la salud $y$ el bienestar, $y$ que pueden tener como consecuencia la expulsión hacia la calle de sus miembros más vulnerables; es decir, niños, niñas $y$ adolescentes.

Este tema surge a raíz de una investigación que se hizo relacionada con la salud sexual y reproductiva de la población adolescente en condición de calle, en la cual se recabó información sobre el tema familiar. De ahí la inquietud por buscar los posibles factores que inciden en el hecho de observar la presencia de menores de edad viviendo en las calles, en albergues (de instituciones públicas o privadas) o bien, en condición de explotación laboral o sexual comercial, a lo que se une la adicción a las drogas $y$ la delincuencia. Se trata de menores que en síntesis, sobreviven en las calles en condiciones de muy alto riesgo.

El rol que juega la familia en la sociedad es determinante en el desarrollo de los individuos, por tal motivo, se tiene como objetivo,

$2 \quad$ Adolescentes que viven en la calle $y$ no tienen ningún vínculo con su familia o bien, adolescentes que están en la calle pero que tienen alguna relación con su familia. conocer las características que presentan las familias de los y las adolescentes en condición de calle, así como tipificar los mecanismos que hacen que ellos y ellas busquen la calle como alternativa. Se denominará a estas familias bajo el concepto de "familias expulsoras", concepto que se puede definir como aquellas familias que expulsan, fuera del hogar, a sus miembros menores de edad, exponiéndolos a todo tipo de atropellos en contra de sus derechos.

Es importante conocer de manera integral, las condiciones en que se desenvuelven las familias dentro de la sociedad, especialmente, aquellas en estado de mayor vulnerabilidad, a fin de comprender los mecanismos que se dan en su interior y que conllevan a la expulsión de las personas menores de edad.

De esta manera, podrían desarrollarse estrategias idóneas en la formulación de las políticas públicas, para abordar de manera más cercana la realidad de estas familias. Estas políticas podrían darse en dos niveles:

1) El de promoción y prevención, con el fin de establecer estrategias que garanticen la permanencia de las personas menores de edad al interior de sus familias en condiciones adecuadas, que les permitan lograr un desarrollo integral; para ello es necesario modificar el ambiente familiar para la reincorporación de la persona menor de edad, en aquellos casos en que se haya dado la expulsión.

2) El de desarrollo de estrategias externas a la familia, que garanticen un desarrollo integral, para el caso de los y las menores de edad que no pueden regresar con sus familias.

Desde esta perspectiva, el Estado - conjunto de instituciones, organizaciones, personas $y$ entes sociales que habitan en un territorio definido - tiene el deber de generar las condiciones estructurales, socio-económicas y culturales que permitan a las y los menores de edad, satisfacer las necesidades básicas y sus derechos para un óptimo desarrollo: alimento, casa, abrigo, familia, salud, aprendizaje y recreación, entre otras. Solamente con la generación de 
condiciones $y$ oportunidades para la atención de estas necesidades, podrán los niños, niñas $y$ adolescentes construir un sentido de respeto por sí mismos y por ende, por los demás; es decir, a partir de que sientan que sus necesidades son atendidas y respetadas, así como que se sientan queridos.

\section{METODOLOGÍA}

Se trata de un estudio descriptivo, por cuanto no se encontraron estudios en Costa Rica, sobre esta temática en particular. Se tiene como objetivo conocer las características que presentan las familias expulsoras.

Las técnicas utilizadas para recoger la información son de carácter cualitativo, basadas en la observación y en entrevistas realizadas a la población meta, en este caso adolescentes en condición de calle, así como sus familiares (cuando fue posible ubicarlos).

\section{A) POBLACIÓN META}

La población meta son los y las adolescentes que se encuentran en condición de calle. Se trabajó con doce participantes en esta condición (siete hombres y cinco mujeres): tres hombres residían en el Albergue La Garita, del Patronato Nacional de la Infancia (PANI), en el momento que se les hizo la entrevista; cuatro mujeres $y$ dos hombres eran atendidos en la Unidad de Atención Inmediata de esta misma institución. Otros dos hombres fueron entrevistados en el Hospital Nacional Psiquiátrico, en donde se encontraban internados por su adicción a las drogas, en el programa Nuevos Horizontes, creado para atender a la población adolescente en condición de calle. Finalmente, una mujer que fue ubicada en la calle y accedió a la entrevista.

\section{B) TRABAJO CON LAS Y LOS ADOLESCENTES}

Con las personas adolescentes se utilizó la técnica de la entrevista semiestructurada, tipo conversatorio, a fin de obtener información que a través de un cuestionario no se obtendría; además, el conversatorio permite que la o las personas entrevistadas amplíen descripciones detalladas de acontecimientos importantes para ellas y que sean relevantes para la comprensión del fenómeno en estudio.

\section{C) TRABAJO CON LAS FAMILIAS}

Por el lugar central que ocupa la familia, uno de los objetivos del estudio fue el de trabajar con los familiares de las personas entrevistadas, a fin de conocer más a fondo sus características y su dinámica. Se consideró la realización de visitas al hogar, lo cual permitiría observar y conocer aspectos del espacio familiar e investigar sobre las características más relevantes de estas. Sin embargo, se tuvo la limitante de que solo se pudo contactar a dos familias, debido a que en algunos casos los participantes no dieron la dirección o lo hicieron de manera incorrecta. En otros casos, no contaban con familia, no tenían ningún contacto con ella o bien, esta se encontraba en zonas alejadas del país. En un caso, se entrevistó al padre del adolescente $y$ en el otro, se entrevistó a la madre $y$ al padrastro de la adolescente.

\section{PAPEL DE LA FAMILIA EN EL DESARROLLO INTEGRAL DE LA PERSONA ADOLESCENTE}

El ser humano es concebido como un ser biopsicosocial, que en la mayoría de los casos, desde su nacimiento, es incluido dentro de una familia. Así, la familia se convierte en el primer ente socializador y el primer contacto de todo ser humano, $y$ como tal, debe garantizar el desarrollo integral de todos sus miembros.

Dentro de este contexto se va a entender el desarrollo integral adolescente, de acuerdo con la Ley de la Persona Joven, en su artículo segundo, como:

...proceso por el cual la persona, mediante el efectivo ejercicio de sus derechos y el acceso democrático a las oportunidades que el Estado garantiza a través de las instituciones, logra un adecuado desarrollo espiritual, social, afectivo, ético, cognoscitivo, físico, moral $y$ material, que la involucre a participar activamente en el desarrollo de la vida nacional y en la identificación y solución 
de los problemas que le afectan como grupo social y a la sociedad como un todo (2002).

En este trabajo, la familia se considera desde una perspectiva sistémica, entendiéndose como sistema a un todo integrado por partes interdependientes. En la aproximación sistémica, se le concibe como un todo, diferente a la suma de las individualidades de sus miembros. Así, se trata de un sistema constituido por una red de relaciones con características propias orientadas a satisfacer las necesidades psicoafectivas de sus miembros. Su función es la de contener a sus integrantes, protegerlos $y$ regularlos con el fin de mantener la estabilidad de este sistema. La familia como tal, se ajusta al concepto de causalidad circular en cuanto que, siendo un grupo de individuos interrelacionados, se ve afectada cuando ocurren cambios a cualquiera de sus miembros.

El grupo familiar, uno de los principales agentes socializadores, se convierte en un medio que de una $u$ otra manera, influye en el individuo, ya sea inhibiendo o favoreciendo su desarrollo, fenómeno que ha sido observado a través de la historia y en las diferentes culturas. Es la familia la que en condiciones ordinarias o "normales", tiene la función más importante en la formación de la identidad; es la fuente de información y de estímulos más determinantes para la estructuración biológica, psicológica y social del individuo. En el desarrollo del proceso de identidad, para el ser humano, son muy importantes las figuras de la madre y del padre, porque se identifica parcial o totalmente con ellas.

La palabra "familia" proviene de la raíz latina famulus, que significa sirviente o esclavo doméstico. En un principio, la familia agrupaba al conjunto de esclavos $y$ criados propiedad de un solo hombre. En la estructura original romana, la familia era regida por el pater quien asumía todos los poderes, incluidos el de la vida y la muerte, no solo sobre sus esclavos sino también sobre sus hijos.

Familia es el nombre con que se ha designado a una organización social tan antigua como la propia humanidad y que simultáneamente, con la evolución histórica, ha experimentado transformaciones que le han permitido adaptarse a las exigencias de cada sociedad y de cada época. Si se analiza a la familia con un enfoque biopsicosocial y con base en la dinámica de las relaciones intrafamiliares, se le puede clasificar en funcional $y$ disfuncional.

Dentro de la familia se debe, en primera instancia, garantizar el interés superior de la persona menor de edad, de los y las adolescentes para el caso específico de este estudio. Se debe garantizar la no discriminación, mantener su condición de sujeto de derecho, así como, la integralidad de estos derechos. El preámbulo de la Convención sobre los Derechos del Niño (aprobada por la Asamblea General de las Naciones Unidas el 20 de noviembre de 1989 y ratificada por Costa Rica en 1990) define a la familia como el grupo fundamental de la sociedad y el medio natural para el crecimiento y el bienestar de todos sus miembros, en particular de los niños, niñas $y$ adolescentes e indica que esta debe recibir la protección y asistencia necesarias para poder asumir plenamente su responsabilidad dentro de la comunidad. Asimismo, reconoce que las personas menores de edad, para el pleno $y$ armonioso desarrollo de su personalidad, deben crecer en el seno de la familia, en un ambiente de felicidad, amor y comprensión. En su art. 5, la Convención sobre los Derechos del Niño manifiesta que los Estados que la suscribieron, respetarán las responsabilidades, los derechos y los deberes de los padres. Es necesario que las sociedades hagan lo posible para evitar que los niños, niñas $y$ adolescentes se separen de su familia (Vicente, 2004).

Por su parte, la Constitución de la República de Costa Rica, establece el derecho de los niños, niñas y adolescentes a tener una familia y disfrutar de la convivencia familiar $y$ comunitaria. El concepto del disfrute de la convivencia familiar, debe llevar a estudiar, exhaustivamente, la situación de las familias y sus reales opciones de garantizar, para las personas menores de edad, un espacio en el cual ellos y ellas puedan disfrutar a plenitud de su vida $y$ encontrar niveles de convivencia con su padre, madre, hermanos, hermanas y demás miembros, basados en el respeto mutuo. 
Es importante destacar que este derecho no solo está planteado en relación con los niños, niñas y adolescentes abandonados, huérfanos, maltratados o abusados sexualmente, sino que se trata de "los derechos para todos los niños, niñas y adolescentes", sin ninguna distinción. Así, es obvio que un derecho fundamental de todas estas personas es vivir con sus padres, no ser separadas de ellos sin causa justa y crecer en un ambiente sano, agradable, caracterizado por una comunicación adecuada, donde haya manifestaciones de amor $y$ de afecto.

La familia debe contar con el apoyo del Estado y la sociedad para que pueda asumir de manera adecuada su rol. Además, hay que tener presente que esta institución social cumple funciones de control muy fuertes, pero también es un espacio en el cual, lamentablemente, se da la violación de los derechos de las personas menores de edad. Como manifiesta Vicente (2004), cuando los niños, niñas y adolescentes hablan del número de castigos semanales que reciben en sus familias $y$ aceptan en un gran porcentaje que está muy bien que les peguen por malcriados, es claro que algo muy grave ha pasado en la conciencia de ellos y ellas. Los cambios que se han producido en la familia, combinados con el grave deterioro de la situación económica y social, están creando ambientes totalmente inadecuados para el desarrollo de las personas menores de edad. Los peligros presentes $y$ potenciales son muy altos, lo que distorsiona las posibilidades de un crecimiento armónico y seguro de las nuevas generaciones.

La crisis económica y social trae como consecuencia, mayor violencia, al propiciar la exclusión social, en especial, de las personas que viven en situación de pobreza. Esto hace que la familia, como un modelo de relación entre seres humanos, no logre cumplir con sus objetivos primordiales.

De acuerdo con Vicente (2004), dentro del seno familiar, las personas menores de edad deben disfrutar de su condición de miembros de la familia, en un marco de respeto a su condición. Así, hay que preguntarse en qué medida los hogares son sitios agradables $y$ acogedores para sus miembros, especialmente, para sus niños, niñas y adolescentes. Se debe analizar si las familias constituyen espacios en los que el amor, la ternura, el respeto y el afecto, superan la obediencia, el control, la sumisión, las exigencias, la humillación, el miedo, las penurias y la agresión, lo que tendería a convertirlas en familias expulsoras.

\section{CONDICIONES SOCIALES Y ECONÓMICAS DE LAS FAMILIAS Y LAS PERSONAS MENORES DE EDAD EN COSTA RICA}

Como se mencionó anteriormente, la familia constituye, para los individuos que la integran, un espacio íntimo en el cual se forman $y$ reproducen los modelos de identidad personal y comunitaria, en el que se aprenden los valores sociales, en el que los niños, niñas y adolescentes se pueden desarrollar integralmente. Sin embargo, es común observar que en muchos casos la familia no cumple con el rol que socialmente se le ha asignado.

Son muchos los factores que afectan el buen desempeño de la familia como ente que debe garantizar el desarrollo integral, siendo los más evidentes las difíciles situaciones socioeconómicas, además de los abusos y la violencia que se cometen contra sus miembros más débiles, por un lado, los niños, niñas y adolescentes, $y$ por otro, las mujeres.

El paradigma de protección integral, que se contempla en la Convención sobre los Derechos del Niño de las Naciones Unidas, establece que las personas menores de edad, además de los derechos contemplados en la Carta Fundamental de los Derechos Humanos, por su condición especial, tienen derechos propios. Esto implica que las relaciones entre los $y$ las menores de edad con la familia, la sociedad y el Estado se modifiquen para una mejor atención a los niños, niñas y adolescentes. Si bien, la relación niñez-adolescencia-familia es respetada en esta convención, el énfasis está puesto en la obligación que tienen el Estado, la sociedad y la familia de garantizar sus derechos.

Dentro de este contexto, es necesario considerar otros factores que inciden, negativamente, para que las personas menores de edad puedan vivir sus derechos a plenitud, como son la reproducción de la pobreza dentro de las familias y la poca movilidad social. Las personas 
menores de 15 años están entre los más afectados por la pobreza y la débil inversión social. Vicente (2004) señala que más de 100 millones de niños en América Latina viven en la pobreza, con todas las consecuencias negativas que esto tiene, a nivel físico y socioafectivo.

En el caso concreto de Costa Rica, de acuerdo con el último censo de población realizado en el año 2011, la población costarricense alcanza los 4301172 habitantes, de los cuales 1310983 (30,5\%) son personas menores de edad. De este grupo, el $21,2 \%$ son niños y niñas y el 9,3\% son personas adolescentes entre los 12 y los 17 años (siedna, 2013). Se trata de un grupo poblacional, que aunque ha tenido un descenso, sigue siendo numeroso, a la vez que presenta características y necesidades diferentes al resto de la población, las cuales, en primera instancia, son responsabilidad de los padres, madres o personas adultas encargadas $y$ en segunda instancia, del Estado.

En lo que se refiere, propiamente a las condiciones socioeconómicas y las políticas sociales que atañen a la población adolescente, se observa que en educación, Costa Rica se caracterizó, en la década de los 90, por el deterioro del sistema educativo público, lo que se refleja en los problemas de cobertura, en la repitencia $y$ en la deserción escolar, en la infraestructura $y$ en la falta de recursos. Esto se contradice con el discurso político imperante de que la educación es un factor protector fundamental para los adolescentes y para combatir la desigualdad social, la pobreza y las inequidades que se generan por las diferencias socioeconómicas.

A pesar de esta situación, de acuerdo con datos del Ministerio de Educación Pública, citados en el iv Estado de los Derechos de la Niñez y la Adolescencia, hay una mejora en la matrícula de estudiantes en todos los niveles, tanto en los centros educativos públicos como privados. Sin embargo, la cobertura tiene un punto crítico en la educación secundaria, tanto es así, que de acuerdo con el "Décimo octavo Informe del Estado de la Nación en Desarrollo Humano Sostenible", con base en datos del censo 2011, la población costarricense tiene un promedio de 8,9 años de escolaridad, a pesar de que la tasa de alfabetización es del 97,6\%. El nivel educativo de la población no aumenta al ritmo que se requiere para un mejor desarrollo personal y social. Por otro lado, se pueden percibir grandes brechas territoriales, dándose una concentración de personas graduadas de secundaria y educación superior en la denominada Gran Área Metropolitana. Existe poco avance en la cobertura de preescolar y el ciclo diversificado $\left(4^{\circ}, 5^{\circ}\right.$ y $6^{\circ}$ años de secundaria), además de que, de acuerdo con datos obtenidos en las pruebas del Programa para la Evaluación Internacional de Alumnos, la calidad de la educación que los estudiantes del país reciben no es la más óptima.

De acuerdo con datos de la Encuesta Nacional de Hogares del 2011, la tasa de escolaridad es alta en los dos primeros ciclos, en donde alcanza casi el 100\% y cubre a niños y niñas de 7 a 12 años. Entre los 2 y los 4 años, solo asiste al sistema educativo el 14\% y el $85 \%$ de los niños y niñas entre los 5 y los 6 años. Para el año 2011, en secundaria, la tasa es de 76,5\%; en el tercer ciclo (tres primeros años de secundaria), esta tasa es de 81, 7\%, en tanto que para el ciclo diversificado, la tasa desciende a 46,5\%. Estas diferencias implican que de cada 10 estudiantes que ingresan al sistema educativo, solo terminan su secundaria aproximadamente 4 personas.

Uno de los principales problemas del sistema educativo es su incapacidad para retener a la población infantil y adolescente en los centros educativos (UCR $y$ unICEF, 2004), a pesar de los esfuerzos que se hacen, como por ejemplo, el programa de becas Avancemos, creado en el 2006. Evidentemente, esto tiene fuertes repercusiones dentro de las familias, pues se da una tendencia de que a menor nivel educativo, menores ingresos y mayor pobreza.

En lo que respecta a la salud, se reconoce la necesidad de establecer un servicio para la población adolescente, así, existe el Programa de Atención Integral del Adolescente (PAIA), el cual "tiene como propósito brindar una atención diferenciada a la población de 10 a 19 años, según conceptos de equidad de género $y$ enfoque de riesgo, para promover, proteger $y$ mantener la salud física, mental y social de los y las adolescentes" (Ministerio de Salud: 1192, citado en UCR y UNICEF, 2004: 40). Sin embargo, 
este programa estuvo a punto de desaparecer y en la actualidad, está muy disminuido.

Costa Rica es un país con buenos índices de salud, en términos generales, aunque con grandes brechas entre grupos sociales $y$ regiones, lo que implica que se requiere de una mejor planificación en lo que a la prestación de los servicios de salud se refiere, teniendo en consideración las necesidades específicas de las distintas zonas geográficas y de los diferentes grupos poblacionales. Sin embargo, el surgimiento de una crisis institucional en la Caja Costarricense de Seguro Social, es un factor de riesgo que podría incidir negativamente en el mantenimiento de los logros en salud, si no se toman las medidas correctivas adecuadas (Programa Estado de la Nación, 2012). Con respecto a las personas menores de edad, se tiene que el $77,1 \%$ es asegurada familiar, el $0,44 \%$ tiene seguro porque son personas asalariadas (por cuenta propia, voluntario o convenio) y se benefician con el régimen no contributivo o reciben alguna pensión; el 11,43\% están aseguradas por el Estado; el 0,73\% tienen otras formas de seguro y el 10,3\% no tiene ningún tipo de seguro. Esta situación genera una serie de cuestionamientos, especialmente si se considera que el $14,5 \%$ de la población del país no cuenta con seguro de la ccss.

La pobreza en los hogares constituye un factor importante, que puede tener como consecuencia que los y las adolescentes abandonen el hogar. En términos generales, se puede decir que:

...la pobreza está asociada a una situación que se caracteriza por severas privaciones que repercuten de manera negativa sobre el desarrollo de las persona $y$ restringen su acceso a un conjunto de bienes y servicios esenciales. Se admite el ingreso o, más precisamente, la insuficiencia de ingreso como una medida adecuada de las deficiencias $y$ dificultades de acceso a los bienes y servicios de infraestructura social (UCR y UNICEF, 2004: 47).

En Costa Rica, la incidencia de hogares pobres, según datos arrojados por el censo 2011 , es de $21,6 \%$, pero si se consideran las personas en vez de hogares, este porcentaje aumenta a $26,5 \%$. El porcentaje de hogares en pobreza extrema es de 6,4\%. Desde el año 1994, el índice de hogares pobres en el país ha tendido a mantenerse en cifras que rondan el $20 \%$, hasta llegar al $21,6 \%$ de acuerdo con la encuesta de hogares del InEc de 2014. Esto quiere decir que el país no ha sido capaz de lograr reducir la pobreza; sin embargo, dado el crecimiento de la población, se ha incrementado el número de hogares en situación de pobreza en los últimos años. En el 2010, el número de hogares pobres era 274616 y 77365 estaban en pobreza extrema; en el 2011, las cifras respectivas son 287367 y 85 557. De acuerdo con cifras de la encuesta de hogares del Instituto $\mathrm{Na}$ cional de Estadística y Censos, esta situación tiende a aumentar, pues el porcentaje de hogares pobres en el año 2014 aumentó a 22,4\% y los que están en pobreza extrema subió a $6,7 \%$. Ello implica un total de 318810 hogares en condición de pobreza y 94810 hogares en pobreza extrema. Este aumento en la pobreza, también implica que casi la cuarta parte de la población costarricense es pobre, pues la cifra alcanza el 24,6\%.

La pobreza implica que el ingreso per cápita en los hogares urbanos es menor a \$92 122 en el área urbana y menor a $\$ 70970$ en la zona rural, ingresos con los cuales no es posible cubrir necesidades básicas. Por su parte, la pobreza extrema en los hogares significa ingresos menores a $\$ 41842$ y a $\$ 34921$, a nivel urbano y rural respectivamente, ingresos con los que no se logra cubrir el costo per cápita mensual de la canasta básica alimentaria. Si a esto se une el indicador de necesidades básicas insatisfechas, los porcentajes aumentan, pues el porcentaje de hogares con algún nivel de necesidades básica insatisfechas es de $24,6 \%$, en tanto que a nivel de población el porcentaje es de $27,4 \%$ (Méndez y Bravo, 2012).

Asimismo, hay una tendencia a que los hogares pobres y especialmente, los que están en pobreza extrema, tengan mayor cantidad de niños menores de cinco años, población mayor de 15 años con menor escolaridad, menor cantidad de ocupados, mayor desempleo y el porcentaje de jefatura femenina más alto. La 
pobreza en hogares encabezados por mujeres alcanza el 37\%.

Por otro lado, se ha dado un incremento de la desigualdad en la distribución del ingreso, lo cual se refleja en el aumento del coeficiente de Gini entre el 2010 y 2011, pasando de 0,508 a 0,515: "La distribución de los ingreso entre los hogares presenta una clara concentración en los grupos de altos ingresos. La relación entre el primer quintil (el $20 \%$ más pobre) y el quinto (el 20\% más rico) aumentó de 16,7 veces en el 2010 a 18,2 veces en el 2011" (Programa Estado de la Nación, 2012:114).

En relación con el empleo, para el 2011, la tasa es de $7,3 \%$. Por quintil, el desempleo del primer quintil es 10 veces mayor que en el quinto quintil: $21,4 \%$ y $2,3 \%$, respectivamente. De acuerdo con la Encuesta Nacional de Hogares 2012, la tasa de búsqueda de nuevos empleos fue de un $5,01 \%$, porcentaje que supera al crecimiento de la población y al crecimiento de la población en edad de trabajar, lo que implica que las condiciones socioeconómicas desfavorables obligaron a más personas a buscar trabajo. También está el hecho de que muchos de los empleos que se obtuvieron fueron en condiciones no deseadas en cuanto a calidad, cantidad y nivel de ingresos. El subempleo se ubica en un $26 \%$ y las mujeres son el grupo más afectado.

Dentro de este panorama en el plano laboral, hay que considerar algunos aspectos como son: un descenso de la población ocupada asegurada directa en el 2011, con respecto al 2010 (se pasó de 71,1\% a 70,6\%, en el caso de las mujeres ocupadas, este porcentaje alcanza el 63,3\%) e incumplimientos en las garantías laborales en el mercado de trabajo, como por ejemplo que uno de cada cinco ocupados no recibe aguinaldo, que tres de cada diez no disfrutan de vacaciones pagadas ni tienen cobertura por enfermedad o riesgos del trabajo y que a casi la mitad no se les reconoce el pago de horas extra (Programa Estado de la Nación, 2012).

En cuanto a la inversión social pública, entre el 2010 y el 2011, disminuyó en un 0,5\%, lo que equivale a una disminución de $1,6 \%$ en términos per cápita (Programa Estado de la Nación, 2012).
Con estos datos, para el año 2011, Costa Rica tiene un nivel de pobreza $y$ de vulnerabilidad del 33,9\%, lo que quiere decir que "ante cambios en la situación económica que afecten los ingresos de los hogares, muchos de ellos muy probablemente caerán en pobreza" (Programa Estado de la Nación, 2012).

Si se mide la pobreza por grupos de edad, en los datos del censo 2011 se puede observar que los porcentajes más altos de pobreza y de pobreza extrema se dan en la población menor de 18 años.

En Costa Rica uno de cada tres niños y adolescentes vive en condiciones de pobreza. Esto equivale a decir que este grupo representa el $45 \%$ de los pobres totales del país. Los menores de 12 años en esta situación representan el $32 \%$ de la población pobre total y el 36,6\% de los pobres extremos. Estas cifras son más relevantes si se considera que solo el 19,1\% de la población nacional es menor de 12 años. En otras palabras, existe una sobrerrepresentación de los infantes en la población pobre, fenómeno que se observa en todas las regiones del país.... Muchos de estos niños y niñas no pueden siquiera cubrir sus necesidades de alimentación, ni acceder a los servicios básicos de salud, vivienda digna, agua potable y electricidad. Si logran incorporarse en el sistema de educación formal - principal mecanismo de movilidad social-, sus posibilidades de mantenerse en él, o de tener un rendimiento exitoso, son escasas. La falta de escolarización temprana y los elevados niveles de reprobación y deserción siguen siendo grandes obstáculos para una integración exitosa de las nuevas generaciones en la sociedad del conocimiento (Programa Estado de la Nación, 2012: 117-118).

Lo anterior evidencia importantes problemas sociales en lo que concierne a la población más joven. Problemas de exclusión, marginalidad y vulnerabilidad de importantes sectores de la población que, en el caso de las personas menores 
de 18 años, se evidencia en un prolongado y sostenido proceso de infantilización de la pobreza (UCR y unICEF, 2004: 50).

Por otro lado, está el factor violencia, que tiende a manifestarse más en las familias que se encuentran en las condiciones descritas. Hoy la violencia se ha llegado a considerar, más que un problema que atañe solo a las políticas de seguridad nacional o de justicia, como un problema de salud pública, dentro de la perspectiva de desarrollo humano integral. Relacionado con este parámetro se observa como en la sociedad surgen pandillas, producto de la falta de planificación urbana, entre otros aspectos, y que llegan a convertirse en la extensión del papel de la familia donde, frecuentemente, los adolescentes encuentran valores como la lealtad, el honor $y$ el compañerismo que nunca encontraron al interior de su propia familia, dada la desintegración familiar $y$ comunitaria, la pobreza $y$ el colapso del modelo de gestión de otra de las instituciones clave para la integración social, como lo es la escuela y el sistema educativo en general, el cual muchas veces se constituye en un ente excluyente, para los adolescentes.

Lo anterior se constituye en condiciones explicativas de las familias expulsoras, sin que eso quiera decir que la expulsión de las personas menores de edad, dependa solo de las condiciones socioeconómicas de las familias. Hay que destacar que estas familias están dentro de un contexto político, económico y social, que se caracteriza por crear grandes desigualdades sociales dentro de los grupos poblacionales $y$ en consecuencia, generar una situación de exclusión social, que se manifiesta, como se señaló anteriormente, en los sectores más pobres de la población.

En estas circunstancias, se produce dentro de la sociedad un círculo vicioso, del que es muy difícil lograr que las familias salgan, por cuanto la pobreza y la desintegración familiar se reproducen si no se formulan medidas integrales generadas dentro de un "orden económico y social radicalmente diferente al actual" (Chávez, 2004: 4), de ahí que hay que tener en cuenta que se pueden formular una serie de recomendaciones para mejorar la situación planteada, que podrían ser medidas que sirvan para paliar la misma y para mejorarla un poco, aunque el problema seguiría persistiendo, a menos que se haga ese cambio radical en la sociedad.

Dentro de este panorama se desarrollan las familias de las personas adolescentes en condición de calle. Es evidente que en condiciones de adversidad, las familias se tornan disfuncionales, en el sentido de que se ven afectados los derechos de sus miembros, especialmente, los menores de edad.

\section{CARACTERIZACIÓN DE LAS FAMILIAS EXPULSORAS}

Con base en las entrevistas realizadas a los y las adolescentes que forman parte de este estudio y a las familias, se encontraron características que evidencian las condiciones adversas a nivel socioeconómico al que se enfrentan, lo que redunda en una serie de problemas a lo interno de las mismas.

A manera de síntesis, las familias en estudio se pueden caracterizar de la siguiente manera:

$\diamond \quad$ Ausencia física o emocional de los progenitores. En algunos casos, estos se encuentran en condición de indigencia.

$\diamond \quad$ Padres no conviven, además no tienen ninguna comunicación entre ellos.

$\diamond \quad$ Los padres delegan la crianza y educación en terceras personas o en instituciones.

$\diamond \quad \mathrm{Al}$ interno de las familias se vive agresión física, agresión verbal y abuso sexual.

$\diamond \quad$ Existe dentro de las familias un manejo inadecuado de normas y límites desde etapas tempranas del desarrollo.

$\diamond \quad$ No brindan contención afectiva.

$\diamond \quad$ Rol de autoridad asumido inadecuadamente por la persona designada.

$\diamond \quad$ Baja escolaridad en los miembros adultos de la familia.

$\diamond \quad$ Baja escolaridad en las personas menores de edad.

$\diamond \quad$ Antecedentes familiares de adicción.

$\diamond \quad$ La preocupación de la persona responsable es satisfacer sus propias necesidades $y$ resolver sus propios problemas, descuidando a los hijos. 
$\diamond \quad$ En la comunidad donde reside la familia, hay fácil acceso a las drogas.

$\diamond \quad$ La familia no fortalece la construcción de una identidad adecuada.

$\diamond \quad$ La atención a la persona menor de edad no fue prioridad de la familia.

$\diamond \quad \mathrm{Al}$ interno de las familias se da el aprendizaje de las conductas inadecuadas.

$\diamond \quad$ No reconocimiento de conductas positivas en las personas menores de edad.

$\diamond \quad$ Reconocimiento limitado de parte de las personas encargadas de la crianza y educación de su cuota de responsabilidad en la problemática de las personas menores de edad.

$\diamond \quad$ No hay comprensión de las manifestaciones de los y las adolescentes cuando niños y niñas, sino que más bien se les reprime $y$ se les castiga física, verbal $y$ emocionalmente.

$\diamond \quad$ La familia no cumplió con su encargo social, no brindo protección a los miembros menores de edad, el proceso de socialización no se realizó de manera adecuada, no protegieron a los muchachos, no les satisfacieron sus necesidades básicas, sus necesidades socio-afectivas.

$\diamond \quad$ No les permitieron enriquecer su identidad a través de la experimentación de las relaciones sentimentales.

$\diamond \quad$ Les violentaron sus derechos como seres humanos, poniéndolos en situación de alto riesgo y convirtiéndolos en personas sumamente vulnerables.

$\diamond \quad$ Estas familias no garantizaron la permanencia de sus hijos dentro del sistema educativo, lo cual no les permitió el proceso socio-afectivo tan importante que se vive tanto en la etapa escolar como en la etapa colegial, limitando así sus posibilidades para enfrentarse al mundo social de una manera adecuada.

$\diamond \quad$ Las personas menores de edad de estas familias no contaron con los espacios necesarios que les permitieran aprender las aptitudes para la vida, básicas en la etapa de la adolescencia cuando se busca la identidad e independencia.

$\diamond \quad$ No se favoreció al interno de estas familias el desarrollo de las potencialidades de sus integrantes, ninguna permitió que ellos lograran alcanzar un desarrollo integral adecuado, por el contrario, se podría afirmar que fue al interior de sus propias familias donde estos adolescentes incorporaron patrones inadecuados de comportamiento, tales como los problemas de adicción, conducta delictiva, abuso sexual, entre otras.

$\diamond \quad$ En dichas familias se detectan factores de alto riesgo (abuso sexual, consumo de drogas y padres indigentes); asimismo, son familias con escasos vínculos entre sus miembros.

$\diamond \quad$ Estas familias son expulsoras de las personas menores de edad, no preparan a sus miembros para su incorporación adecuada a la sociedad, por el contrario, los expulsan hacia el afuera, donde los sitúa en condiciones de muy alto riesgo. Los expone a experimentar la explotación laboral, la explotación sexual comercial, la pornografía infantil, el trasiego y el consumo de drogas, el tráfico internacional y nacional, el robo y la mendicidad, como formas de sobrevivir en este mundo.

$\diamond \quad$ Falta de credibilidad dentro del grupo familiar cuando la persona menor de edad quiere decir que está sufriendo abusos por parte de alguno de los adultos de la familia.

\section{CONCLUSIONES}

Como se señaló anteriormente, hay que ubicar a las familias de la población adolescente en condición de calle dentro de un contexto social, político y económico que tiende a ensanchar la brecha social y que genera pobreza. Esto ubica a estas familias en condiciones adversas, con las consiguientes consecuencias en la dinámica interna de las mismas, en perjuicio de las personas menores de edad.

La caracterización anterior muestra la tendencia a que las familias de las y los adolescentes entrevistados no cumplieron con su encargo social, no brindó protección a los miembros menores de edad, no les proporcionó una socialización adecuada, no les satisfacieron sus 
necesidades básicas y sus necesidades socioafectivas, no les permitieron enriquecer su identidad a través de la experimentación de las relaciones sentimentales, les violentaron sus derechos como seres humanos, poniéndolos en situación de alto riesgo y convirtiéndolos en personas sumamente vulnerables.

Estas familias no garantizaron la permanencia de sus hijos dentro del sistema educativo, limitando así sus posibilidades para enfrentarse al entorno social de una mejor manera. No contaron con los espacios necesarios que les permitieran aprender las aptitudes para la vida, básicas en la etapa de la adolescencia, cuando buscan la identidad e independencia.

No se favoreció a lo interno de estas familias el desarrollo de las potencialidades de sus integrantes, ninguna permitió que los adolescentes lograran alcanzar un desarrollo integral, más bien, se puede afirmar que fue al interior de sus propias familias donde ellos incorporaron patrones de comportamiento de riesgo, como son la adicción y la conducta delictiva, entre otras.

En dichas familias se detectan factores de alto riesgo al interior de las mismas (abuso sexual, consumo de drogas, padres indigentes, entre otros), son familias con pobres vínculos entre sus miembros $y$ hay evidencia de violencia intrafamiliar.

Estas familias son expulsoras de las personas menores de edad, no preparan a sus miembros para su incorporación adecuada a la sociedad, los sitúa en condiciones de muy alto riesgo, los expone a experimentar diversos peligros como formas de sobrevivencia.

Esto trae como consecuencia, a largo plazo, que estas personas que hoy son menores de edad, no se encuentran preparados para llegar a formar una familia, porque no lo han aprendido y es muy probable que si no reciben el apoyo necesario y apropiado, reproduzcan el rol expulsor de sus familias de origen, ya que bajo los esquemas de convivencia que han tenido, se les define un carácter desconfiado, inseguro, sin proyección hacia el futuro; es decir, carentes de metas y objetivos.

Carecen de estructuras emocionales y lazos afectivos, lo que llega a formar parte intrínseca de su personalidad $y$ de su vida. Se transforman así en víctimas y reproductores de un sistema marginal. No hay comprensión de las manifestaciones de los adolescentes cuando niños, sino que más bien se les reprime y se les castiga físicamente.

La satisfacción de las necesidades en etapas anteriores a la adolescencia, cobra mucha relevancia, si se considera que la adolescencia es una etapa importante en el desarrollo humano, en donde se observa con mayor intensidad los aprendizajes psicosociales que se han generado en esas etapas anteriores, el desarrollo intelectual $y$ el desarrollo de destrezas, habilidades y apropiación de roles, aspectos que en constante interrelación le permiten al ser humano un enriquecimiento personal y una mejor convivencia social, garantizando así un desarrollo integral. En las situaciones analizadas se ve como todos estos procesos han sido interrumpidos, lo que lleva a decir que ninguna familia de las estudiadas ha permitido que sus integrantes menores de edad se desarrollen de la mejor manera.

\section{RECOMENDACIONES}

De acuerdo a los resultados obtenidos en el presente estudio, se formulan las siguientes recomendaciones:

$\diamond \quad$ Recuperar la centralidad de la familia como la unidad básica para la protección y la socialización de niños, niñas y adolescentes, apoyando la prevención de su desintegración por medio de legislaciones que le otorguen la prioridad que merece en inversión social y en el diseño de programas de asistencia económica, capacitación laboral y educación para prevenir la violencia intrafamiliar y la expulsión de niños, niñas y adolescentes de sus hogares.

$\diamond \quad$ Desarrollar campañas de salud sexual y reproductiva con énfasis en la maternidad y paternidad deseada y responsable.

$\diamond \quad$ Capacitar a padres cuyos hijos inician el proceso educativo formal, sobre las mejores prácticas de apoyo en la vida escolar. 
$\diamond \quad$ Realizar proyectos comunitarios para facilitar el análisis de la maternidad y paternidad dentro de un proyecto de vida, de manera que las personas menores de edad puedan visualizar opciones sobre su vida adulta asociadas a tener o no tener hijos.

$\diamond \quad$ Capacitar a futuros padres sobre formas efectivas $y$ protectoras de crianza: cuido diario, establecimiento de límites desde el nacimiento, etc.

$\diamond \quad$ Fortalecer el valor de la vida en familia.

$\diamond \quad$ Brindar acompañamiento $y$ "empoderamiento" a las familias que presentan condiciones de riesgo, que les permita fortalecer su capacidad para atender a sus hijos e hijas y garantizar sus derechos en el máximo de sus posibilidades.

$\diamond \quad$ Considerar el espacio comunitario, el cual es el lugar en el que se encuentran posibilidades, tanto naturales como cotidianas, para la socialización y contención afectiva, así como oportunidades para la recreación y la educación. Así, se requiere crear ambientes comunitarios saludables, "que respondan a las necesidades de desarrollo de los jóvenes, que permitan su inclusión social y su participación protagónica, que ofrezcan alternativas diversas para la satisfacción de sus necesidades y el ejercicio de sus deberes" (Rodríguez, Garita, Díaz y Sequeira, 2002: 82).

Para operacionalizar este nuevo enfoque, se requerirá de un proceso de reflexión, sensibilización y capacitación del personal que labora en contacto directo con las familias, para poder fortalecer su capacidad para mantener una posición técnica y una actitud positiva y esperanzadora aún en las situaciones más difíciles. Desde esta perspectiva $y$ la de los derechos de los niños, niñas y adolescentes, lo más urgente, es que desde los diferentes ámbitos en que se brindan servicios a la niñez y adolescencia, se garantice su permanencia en familias fortalecidas capaces de ofrecer condiciones adecuadas para su desarrollo, desde el contexto cultural, étnico y social en que estas se encuentren.
Cabe destacar que dentro de esta perspectiva, se pueden distinguir tres niveles:

1) Nivel primario: acciones de educación y promoción a nivel nacional, regional y local sobre los derechos y responsabilidades de la población menor de edad, propiciando entre otras cosas, el desarrollo integral $y$ un cambio de cultura respecto a la concepción que se debe de tener sobre esta población como sujeto de derechos, así como, la relación que deben establecer las personas menores de edad con las personas adultas y viceversa. El trabajo a este nivel cubre al total de la población menor de edad que habita en Costa Rica $y$ a sus familias, lo que hace prioritario que los beneficios son invaluables a largo plazo; si los esfuerzos son bien canalizados, la probabilidad de que surjan condiciones de riesgo es mínima.

2) Nivel secundario: el nivel secundario implica el conjunto de acciones preventivas dirigidas hacia las personas menores de edad que sufren algún nivel de riesgo $y$ hacia sus familias. Implica identificar aquellos niños, niñas y adolescentes que se encuentran en situación de riesgo por no tener acceso a servicios, por haber dejado el sistema educativo o por estar involucrados en comunidades o familias donde impera la violencia, la drogadicción u otras problemáticas. No obstante, en este nivel las familias aún tienen posibilidades, con el debido apoyo, de mantener a sus hijos e hijas bajo su responsabilidad. Incluye las situaciones de familias adolescentes, madres adolescentes que tienen algún apoyo o recurso, personas con discapacidad que cuentan con familias, poblaciones en lugares distantes 0 que por condiciones culturales no tienen acceso a los diferentes servicios que se ofrecen en la sociedad.

3) Nivel terciario: acciones reivindicativas de derechos a personas menores de edad que sufren situaciones violatorias de sus derechos y que incluyen tanto su atención directa como su protección de personas 
menores de edad que ya enfrentan condiciones severas de problemática familiar, incluyendo abandono, maltrato o abuso de cualquier índole: físico, emocional, sexual, trabajo infantil, explotación laboral, explotación sexual comercial, violencia juvenil, conflicto con la ley, pandillas juveniles, discapacidad y abandono, madres adolescentes sin apoyo, drogadicción del niño(a) o joven y otras situaciones semejantes en gravedad.

Se trata de establecer políticas públicas que permitan una atención integral y no meramente asistencialista a las personas menores de edad que se encuentran en condición de calle $y$ a las familias que se encuentran en condiciones sociales, económicas y culturales adversas, que las caracterizan como familias expulsoras.

\section{BIBLIOGRAFÍA}

LIBROS

Krauskopf, Dina. Participación social y desarrollo en la adolescencia. Tercera Edición. San José, Costa Rica. 2003.

León, Ana Teresa. El maestro y los niños. La humanización del aula. San José, Costa Rica: Editorial de la Universidad de Costa Rica, 2002.

\section{TESIS}

Castro, Ana Cecilia y Prieto, Olga. "Salud sexual y reproductiva de la población adolescente en condición de calle: un estudio de casos, Patronato Nacional de la Infancia, julio-setiembre de 2009". [Trabajo final de investigación aplicada para optar por el grado de Magister en Salud Pública con énfasis en Adolescencia y Juventud]. Universidad de Costa Rica, 2009.

OTROS

Chávez Frías, Hugo. "En apoyo a la propuesta de Lula: hambre cero. ¿Queremos acabar con la pobreza? Demos poder a los pobres". Reunión de Jefes de Estado convocada por el Presidente de Brasil Luiz Inácio Da Silva. Naciones Unidas, setiembre 2004.

Consejo Nacional de la Política Pública de la Persona Joven. Politica Pública de la Persona Joven. Documento de Trabajo para la Asamblea Nacional de la Red Consultiva. Setiembre, 2003.

Constitución Política de la República de Costa Rica. 7 de noviembre de 1949.

Fondo Internacional de Emergencia de las Naciones Unidas para la Infancia-uniceF. Estado mundial de la infancia. 2002.

Instituto Nacional de Estadística y Censo (INEC). Encuesta Nacional de Hogares. Cifras básicas sobre fuerza de trabajo, pobreza e ingresos. San José, Costa Rica, julio 2011.

Instituto Nacional de Estadística y Censo (INEC). Encuesta Nacional de Hogares Julio 2012. Resultados Generales. San José, Costa Rica: Inec, 2012.

Instituto Nacional de Estadística y Censo (INEC). Encuesta Nacional de Hogares Julio 2014. Resultados Generales. San José, Costa Rica: Inec, 2014.

Instituto Nacional de Estadística y Censo (INEC). $X$ Censo Nacional de Población y VI de vivienda 2011. Resultados generales. San José, Costa Rica: Inec, mayo 2012.

Ley General de la Persona Joven. Ley Nro. 8261. 2002.

Mauras, Marta. "La familia y las políticas públicas: hacia una 'sociedad de redes". Politicas hacia las familias, protección e inclusión sociales. Documento de la reunión de expertos de la cepal. Chile, junio 2005. En: <http://www.cepal. org/dds/noticias/paginas /2/21682/ Marta_Mauras.pdf>

Méndez, Floribel y Bravo, Odilia. "Mapas de pobreza con datos censales". Ponencia presentada en el Simposio Costa Rica a la luz del Censo 2011. Universidad de Costa Rica, octubre 2012.

Ministerio de Educación. Estadísticas en Educación, 2011. Departamento de Estadística, Ministerio de Educación, 2014. 
Programa Estado de la Nación. Décimo octavo Informe del Estado de la Nación en Desarrollo Humano Sostenible, 2012. San José, Costa Rica: Programa Estado de la Nación, 2012.

Proyecto Sistema de Información Estadística en Derechos de la Niñez y la Adolescencia (siedna). Personas menores de edad a la luz del censo 2011. San José, Costa Rica: Instituto Nacional de Estadística y Censo-Inec, Universidad de Costa Rica-ucr, marzo 2013.

Rodríguez, Julieta; Garita, Carlos; Díaz, Marco y Sequeira, Mynor. Bases Programáticas. Programa de Atención Integral a la Adolescencia. Oscar Valverde Cerros (consultor). Caja Costarricense de Seguro Social-ccss, 2002.

Universidad de Costa Rica y unICEF. III Informe del Estado de los derechos de la niñez y la adolescencia en Costa Rica. Universidad de Costa Roca-ucr, Fondo Internacional de Emergencia de las Naciones Unidas para la Infancia-unicef, 2002.
Universidad de Costa Rica y unicef. iv Informe del Estado de los Derechos de la Niñez y la Adolescencia en Costa Rica. Universidad de Costa Rica, 2004.

Universidad de Costa Rica y unICEF. VII Informe del Estado de los Derechos de la Niñez y la Adolescencia en Costa Rica. Universidad de Costa Rica-ucr, Fondo Internacional de Emergencia de las Naciones Unidas para la Infancia-unicef, 2012.

Vicente Salazar, Rodolfo. La familia. Documento interno. Costa Rica: Patronato Nacional de la Infancia-pani, 2004.

Vicente Salazar, Rodolfo. La familia y el gozo de vivir. (Artículo inédito) sin fecha.

Vicente Salazar, Rodolfo. La familia desde el enfoque de derechos. (Artículo inédito) sin fecha.

Fecha de ingreso: $23 / 03 / 2015$ Fecha de aprobación:24/04/2015 